\title{
Bilateral tension pneumothorax during endoscopic submucosal dissection under general anesthesia diagnosed by point-of-care ultrasound - A case report -
}

\section{Seok Kyeong Oh, Seung Inn Cho, Young Ju Won, and Jin Hee Yun}

Received November 3, 2020

Revised January 19, 2021

Accepted January 21, 2021

\section{Corresponding author}

Seok Kyeong Oh, M.D., Ph.D.

Department of Anesthesiology and

Pain Medicine, Korea University Guro

Hospital, 148 Gurodong-ro, Guro-gu,

Seoul 08308, Korea

Tel: 82-2-2626-1437

Fax: 82-2-2626-1438

E-mail: nanprayboy@korea.ac.kr
Department of Anesthesiology and Pain Medicine, Korea University Guro Hospital, Korea University College of Medicine, Seoul, Korea

Background: Endoscopic submucosal dissection has become popular. However, this can cause serious complications. In this case, esophageal perforation caused bilateral tension pneumothorax.

Case: A 60-year-old man with esophageal adenoma underwent endoscopic submucosal dissection under general anesthesia. The peak airway pressure was $25 \mathrm{cmH}_{2} \mathrm{O}$ after induction but abruptly increased to $40 \mathrm{cmH}_{2} \mathrm{O}$ after $30 \mathrm{~min}$. Respiratory sounds were barely heard. The lack of lung sliding in either (right-dominant) lung on ultrasound. Within minutes, oxygen saturation and systolic blood pressure decreased to $52 \%$ and $70 \mathrm{mmHg}$. Emergent needle thoracostomy, followed by chest tube insertion, was performed on right chest and his vital signs stabilized. Upon transfer to intensive care unit, oxygen saturation and blood pressure decreased again; therefore, a left chest tube was inserted.

Conclusions: Pneumothorax due to esophageal perforation can lead to life-threatening tension pneumothorax. Anesthesiologists should be aware of the risks and emergency treatment. Ultrasound can be useful for immediate bedside patient-care decisions.

Keywords: Diagnostic ultrasound; Endoscopic gastrointestinal surgical procedures; Endoscopic mucosal resection; General anesthesia; Tension pneumothorax.
Endoscopic submucosal dissection (ESD) is a popular esophageal-sparing and minimally invasive treatment for superficial esophageal carcinoma [1]. Successful ESDs require minimizing patient movement for good visualization as well as for managing the patients' general condition [2]. In Korea and Japan, this procedure is usually performed under conscious sedation in endoscopy rooms [2]. However, in cases with lesions in locations that are difficult to approach and patient noncompliance leading to patient movement, ESD can be performed under general anesthe- sia in an operating room $[2,3]$.

ESD was developed to overcome the limitations of conventional endoscopic mucosal resection (EMR) caused by incomplete resection [4]. Meta-analyses reported higher en bloc and curative resection rates along with a higher risk of perforation and longer operation times due to large wound incidence and difficulties with ESD compared to that with EMR $[5,6]$. In addition, compared to endoscopic gastric procedures, endoscopic esophageal procedures have a higher risk of perforation because of the thin esophageal

This is an Open Access article distributed under the terms of the Creative Commons Attribution Non-Commercial License (http://creativecommons.org/licenses/by-nc/4.0) which permits unrestricted non-commercial use, distribution, and reproduction in any medium, provided the original work is properly cited.

Copyright (c) the Korean Society of Anesthesiologists, 2021 
wall and motion due to respiration and heartbeat in a narrow space [7]. Therefore, precise expertise and skills are required to perform ESD.

Esophageal perforation is a complication that an anesthesiologist may encounter during the procedure. The reported esophageal perforation rate during ESD is approximately $4.5 \%[3,4,6]$. This complication is managed intraoperatively by clip closure [8]. However, if the perforation is not managed properly, it can progress to subcutaneous or mediastinal emphysema or pneumothorax.

In our case, esophageal perforation led to bilateral tension pneumothorax and subcutaneous emphysema, resulting in hemodynamic instability during the procedure. As ESD is increasingly being performed under general anesthesia, this study is essential as it describes the potentially life-threatening complications.

In an emergency situation, it is important to promptly detect life-threatening complications to minimize the delay between symptom onset and initiation of definitive therapy. These goals can be achieved using point-of-care ultrasound. Point-of-care ultrasound is helpful in the diagnosis of pneumothorax [9]. We report a case of bilateral tension pneumothorax during endoscopic submucosal dissection under general anesthesia, which was diagnosed using point-of-care ultrasound. This report highlights a clinical case of acute care in which bedside ultrasound can be used as a crucial diagnostic tool.

\section{CASE REPORT}

A 60-year-old man diagnosed with esophageal adenoma (high-grade dysplasia) was scheduled for ESD under general anesthesia. He was $169.9 \mathrm{~cm}$ tall and weighed $69.2 \mathrm{~kg}$. He had underlying hypertension, with normal preoperative laboratory and chest radiography findings. He had no history of pulmonary disease.

As premedication, $0.2 \mathrm{mg}$ mobinul and $2 \mathrm{mg}$ midazolam were administered intramuscularly. His initial vital signs before anesthesia were as follows: blood pressure (BP), 170/100 mmHg; heart rate, 100 beats/min; and peripheral oxygen saturation $\left(\mathrm{SpO}_{2}\right), 99 \%$. As induction agents, 150 $\mathrm{mg}$ propofol, $50 \mathrm{mg}$ rocuronium bromide, and $50 \mu \mathrm{g}$ fentanyl were administered. Mask ventilation was provided for 3 min with $8 \mathrm{~L} / \mathrm{min}$ oxygen for preoxygenation, and intubation was performed with an 8.0-mm endotracheal tube. Anesthesia was maintained with 6 vol\% desflurane, $1.5 \mathrm{~L} /$ $\min \mathrm{N}_{2} \mathrm{O}$, and $1.5 \mathrm{~L} / \mathrm{min}_{2}$. Mechanical ventilation was set at pressure control and volume guarantee mode with a tidal volume of $600 \mathrm{ml}$ and a respiratory rate of 12 beats per min. During the procedure, the patient was placed in the left lateral decubitus position, and his peak inspiratory airway pressure (PIP) was maintained at $25 \mathrm{cmH}_{2} \mathrm{O}$.

The patient's PIP suddenly increased to $35 \mathrm{cmH}_{2} \mathrm{O} 30$ min after the operation. His vital signs were as follows: BP, 160/110 mmHg; heart rate, 80 beats/min; $\mathrm{SpO}_{2}, 100 \%$; and end-tidal carbon dioxide, $27 \mathrm{mmHg}$. To decrease the PIP, the tidal volume setting was reduced to $500 \mathrm{ml}$ from 600 $\mathrm{ml}$; however, the PIP increased to $40 \mathrm{cmH}_{2} \mathrm{O}$ within seconds. The endotracheal tube was confirmed to be intact but extensive subcutaneous emphysema was observed around the neck and chest areas. $\mathrm{N}_{2} \mathrm{O}$ was stopped and 8 $\mathrm{L} / \mathrm{min} \mathrm{O}_{2}$ was supplied using a fraction of inspired oxygen $\left(\mathrm{FiO}_{2}\right)$ of 1.0. The operator suspected a mid-esophageal perforation. Within a few minutes, the patient's actual tidal volume had decreased to $<100 \mathrm{ml}$.

The surgeon stopped the procedure and repositioned the patient in the supine position from the lateral decubitus position. Breathing sounds were barely heard in both lungs during manual ventilation. Portable chest X-ray (CXR) and arterial blood gas analysis showed the following: $\mathrm{pH}, 7.22$; $\mathrm{PaCO}_{2}$, $61 \mathrm{mmHg} ; \mathrm{PaO}_{2}, 86 \mathrm{mmHg} ; \mathrm{HCO}_{3}^{-}, 25.0 \mathrm{mmol} / \mathrm{L}$; and $\mathrm{SaO}_{2}, 94 \%$ for $\mathrm{FiO}_{2}$ of 1.0. While waiting for the CXR to be uploaded (approximately 10-20 min in our center), the patient's lung was scanned using ultrasound (Sonosite Edge, FUJIFILM Sonosite Inc., USA) with a multi-frequency linear array transducer probe (HFL38x, 13-6 MHz, $6 \mathrm{~cm}$, FUJIFILM Sonosite Inc.). The lung sliding sign (to-and-fro movement of the pleural line) was not observed in the B-mode and parallel lines, termed barcode or stratosphere sign, were identified in the M-mode at the right third intercostal space and mid-clavicular line, indicating a suspected pneumothorax (Fig. 1). Additionally, lung pulse and B-lines were absent and real images, such as consolidation or effusion, were not present in the sonographic evaluation. Despite the presence of broad subcutaneous emphysema, the emphysema depth did not significantly hinder identification on the sonographic image. However, the lung point was not clearly identified with the probe sliding laterally and inferiorly.

Even with a $\mathrm{FiO}_{2}$ of 1.0 before ultrasound scanning, the patient's $\mathrm{SpO}_{2}$ decreased and facial cyanosis was apparent. Within minutes, his $\mathrm{SpO}_{2}$ decreased to $52 \%$, and his systolic BP decreased to $70 \mathrm{mmHg}$. Immediately after this vital sign instability, an 18-gauge needle was inserted into the right 
second intercostal space at the mid-clavicular line for needle thoracostomy, and an emergency doctor was called for chest tube insertion. Immediately after needle thoracostomy, the patient's $\mathrm{SpO}_{2}$ and systolic $\mathrm{BP}$ increased to $96 \%$ and $100 \mathrm{mmHg}$, respectively. The CXR was uploaded after nee-

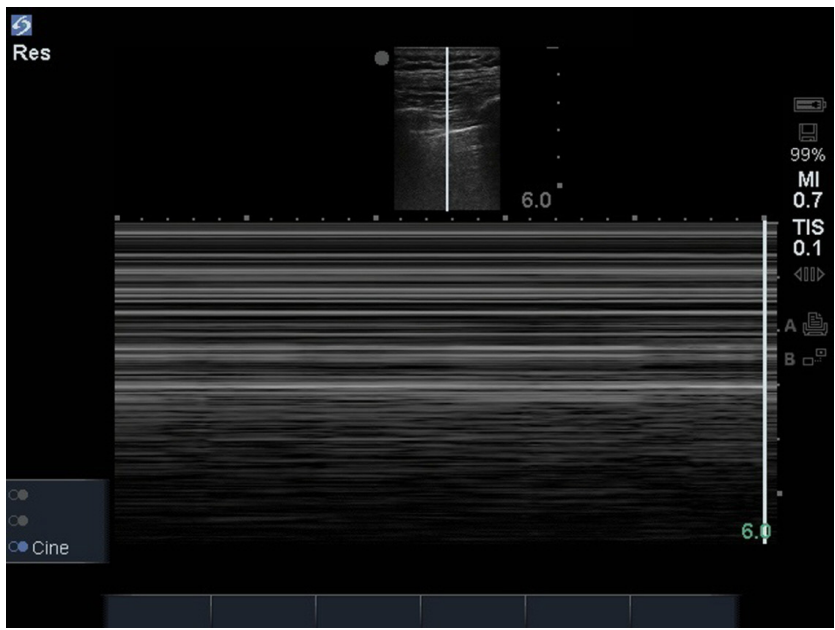

Fig. 1. Lung ultrasound. Upper: B-mode. Lower: M-mode. The normal homogenous granular pattern generated by the lung sliding (lung sliding sign) is not visible, representing the static chest wall (barcode or stratosphere sign). dle thoracostomy and the diagnosis of bilateral (right-dominant) tension pneumothorax was confirmed (Fig. 2). After chest tube insertion on the right side by the emergency doctor, the patient's vital signs stabilized within minutes. As the patient was stabilized, the medical providers decided to transfer the patient promptly to the intensive care unit for further evaluation and management. Using the endotracheal tube, the patient was ventilated with an Ambu bag with oxygen supply during transfer. However, while being transported to the intensive care unit, his $\mathrm{SpO}_{2}$ and systolic BP decreased to $89 \%$ and $80 \mathrm{mmHg}$, respectively. A left chest tube was inserted while the patient was in the intensive care unit, and his vital signs recovered soon after. Three days later, video-assisted thoracoscopic esophagectomy and esophagogastrostomy were performed. After a successful operation, the patient was discharged without further complications.

The patient provided written informed consent for the publication of his information. Ethical permission from the local ethics committee was not required because this case focused on clinical care with general anesthesia and did not involve any human studies.
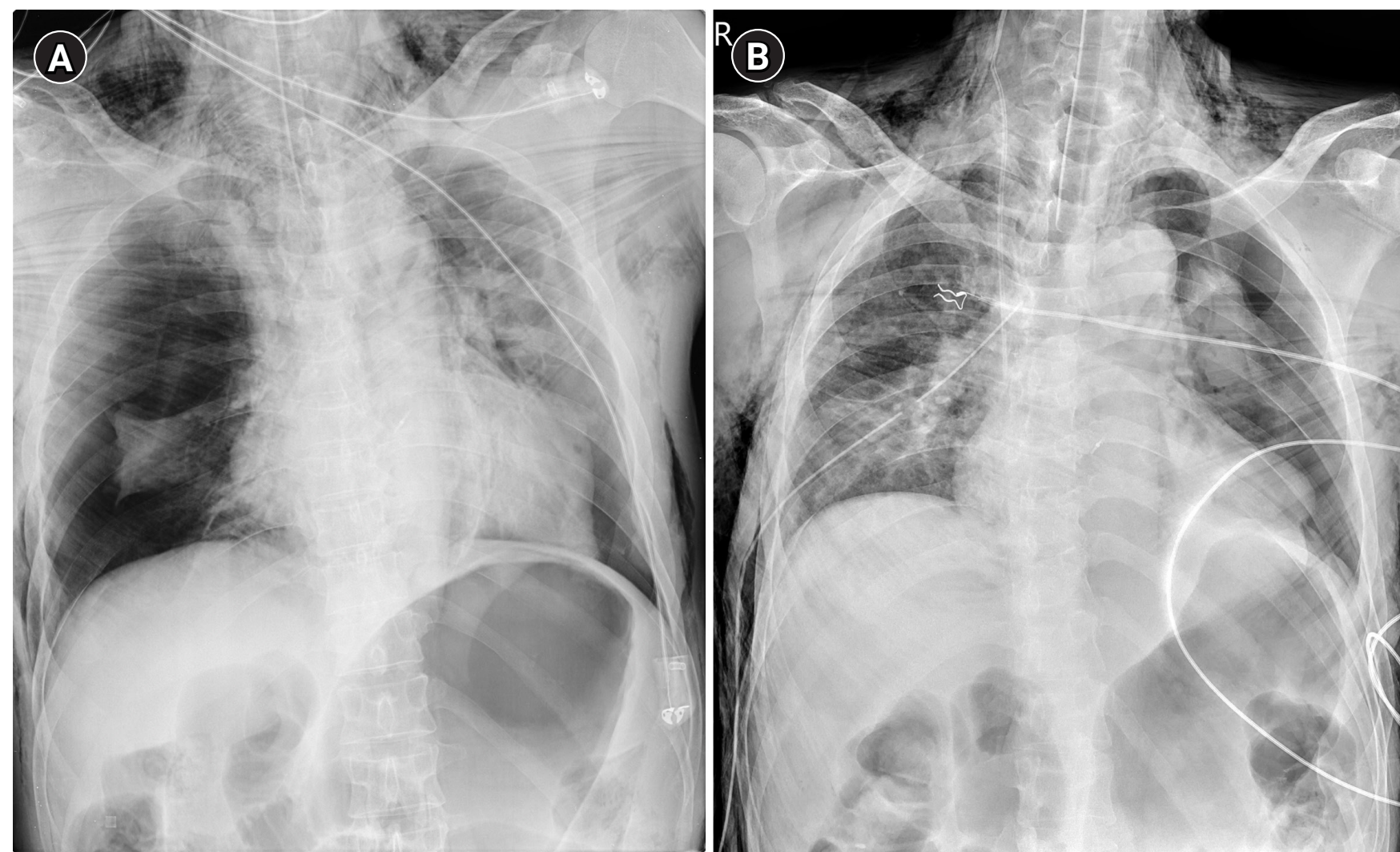

Fig. 2. Chest radiographs. (A) Intraoperative chest radiograph showing bilateral (right-dominant) tension pneumothorax. (B) Chest radiograph on postoperative day 3 showing the resolved pneumothorax with an inserted chest tube. 


\section{DISCUSSION}

While previous reports have described tension pneumothorax during ESD, reports on tension pneumothorax during general anesthesia are scarce. In addition, although the usefulness of point-of-care ultrasound in emergency situations due to complications such as tension pneumothorax is well known, its application to anesthetized patients in an operating room has not been sufficiently reported. This case was an unfamiliar and unexpected situation encountered by an anesthesiologist. Although ESD has been widely accepted for the treatment of esophageal cancer, applying general anesthesia for ESD is not a common practice yet. Studies have shown that general anesthesia during ESD yields better clinical outcomes than those with conscious sedation $[1,3]$. The reported perforation rate during ESD is lower in patients receiving general anesthesia than that in patients receiving conscious sedation with propofol (1.2\% vs. $14.0 \%$ ) [3]. Thus, ESD under general anesthesia has the potential to become more popular.

Perforation during endoscopy can be managed using endoscopic closure with endoclips [10]. However, in this case, esophageal perforation led to bilateral tension pneumothorax, resulting in a devastating situation. General anesthesia was used in this case due to the difficulty of approaching the lesion, which is associated with a high risk for esophageal perforation due to the cumbersome procedure. A consideration of the high perforation risk is essential in patients with such lesions. Patients should always be informed of the risk of perforation and subsequent pneumothorax before ESD, and physicians should be aware of its signs and be prepared for emergency treatment. Positive pressure ventilation during general anesthesia is associated with rapid pneumothorax progression. In addition to positive pressure ventilation, the use of $\mathrm{N}_{2} \mathrm{O}$ in this case, which can cause a rapid increase in the size of a preexisting pneumothorax, might have been a factor in the rapid pneumothorax progression, although $\mathrm{N}_{2} \mathrm{O}$ administration was stopped after confirming extensive subcutaneous emphysema.

In the present case, pneumothorax as a subsequent complication following perforation was diagnosed and managed appropriately. In the diagnosis of tension pneumothorax, physical examination alone has shown poor sensitivity (4.7-17.9\%) [11]; thus, its combination with radiographic findings is essential. Although radiographic findings showed a positive likelihood ratio of 83.7 , with a sensi- tivity of $50.2 \%$ and specificity of $99.4 \%$, the sensitivity of ultrasound is considerably higher (90.9\%) with comparable specificity (98.2\%) [12]. Furthermore, an advantage of ultrasound is its real-time accessibility. In this case, bedside detection using ultrasonography was performed immediately after the event [13]. However, it would have been more appropriate to use needle thoracotomy immediately after ultrasound as the first diagnostic tool of choice before CXR or arterial blood gas analysis. Prompt chest evaluation by point-of-care ultrasound may be helpful in the diagnosis of pneumothorax, especially when time is critical.

For appropriate ultrasound-aided pneumothorax diagnosis and management, medical providers need to be trained and prepared to perform lung ultrasound. The identification of pneumothorax by lung ultrasound with a high level of confidence requires the following four sonographic signs: (i) the presence of lung point, (ii) absence of lung sliding, (iii) absence of B-lines, and (iv) absence of lung pulse [14]. The latter three signs were identified, but the first sign, the lung point, was not observed in our case. In the diagnosis of pneumothorax, the lung point is considered a pathognomonic sign, and only in cases with absence of lung sliding without the lung point, other diagnostic modalities are required $[9,13]$. In patients who are unstable or in cardiac arrest, the conventional methodology with a step-by-step procedure for the detection of the four ultrasound signs varies slightly. In emergency situations with unstable patients, as in our case, the absence of the latter three signs (sliding, pulse, and B-lines) is enough to confirm the diagnosis and move to immediate implementation of life-saving invasive treatment [15]. The lung point is where the visceral and parietal pleural surfaces are divided; that is, the point at which the pneumothorax ends. In the case of massive tension pneumothorax, since pneumothorax has occurred over the entire area, lung sliding is not visible in all areas in lung sonography; thus, the lung point will not be observed. In a life-threatening emergency, there is no need to waste time to check for the presence of a lung point as its visualization would not change the choice of treatment. Even in the absence of the lung point, tension pneumothorax can be diagnosed and should be treated immediately; however, pneumothorax still requires treatment even when the lung point is seen [15].

Pneumothorax defects involving $<20 \%$ of the hemithorax in patients who are clinically asymptomatic and hemodynamically stable can be managed by supplemental oxygen and observation. However, pneumothorax in patients 
with defects involving $>20 \%$ of the hemithorax or symptoms should be decompressed with a chest tube [16]. Patients with bilateral tension pneumothorax show severe dyspnea, cyanosis, or tachycardia that result in decreased cardiac output due to decreased venous return owing to the compression of the vena cava, right atrium, or large veins, which may be life-threatening [17]. Thus, emergent needle decompression is required, which serves as a bridge until chest tube thoracostomy is provided as a definitive treatment. Medical providers must be familiar with the procedure and its potential complications.

As the patient's vital signs stabilized after decompression on the right (dominant) side of the lung, the patient was transferred to the intensive care unit. However, since positive pressure ventilation was performed during the transfer and was planned in the intensive care unit, the possibility of pneumothorax progression on the left side had to be considered. Therefore, it would have been better to have performed decompression on the left side before the transfer.

In conclusion, pneumothorax due to esophageal perforation can lead to life-threatening tension pneumothorax.

Anesthesiologists should be aware of the risks associated with emergency treatment. In addition, point-of-care ultrasound is a crucial tool for early bedside diagnosis in an acute setting. The findings of this report suggest that it is essential for all anesthesiologists to become proficient in clinically integrated point-of-care whole-body ultrasound, including lung ultrasound, for the early diagnosis and prompt treatment of all life-threatening emergencies related to minimally invasive procedures.

\section{CONFLICTS OF INTEREST}

No potential conflict of interest relevant to this article was reported.

\section{DATA AVAILABILITY STATEMENT}

The datasets generated during and/or analyzed during the current study are available from the corresponding author on reasonable request.

\section{AUTHOR CONTRIBUTIONS}

Conceptualization: Seok Kyeong Oh. Supervision: Young Ju Won. Writing - original draft: Seung Inn Cho, Seok Kyeo- ng Oh. Writing - review \& editing: Seok Kyeong Oh, Jin Hee Yun.

\section{ORCID}

Seok Kyeong Oh, https://orcid.org/0000-0002-2939-1848

Seung Inn Cho, https://orcid.org/0000-0002-8375-8786

Young Ju Won, https://orcid.org/0000-0003-4046-0020

Jin Hee Yun, https://orcid.org/0000-0002-4078-8678

\section{REFERENCES}

1. Hamada K, Kawano K, Yamauchi A, Koyanagi R, Horikawa Y, Nishida S, et al. Efficacy of endoscopic submucosal dissection of esophageal neoplasms under general anesthesia. Clin Endosc 2019; 52: 252-7.

2. Yamashita K, Shiwaku H, Ohmiya T, Shimaoka H, Okada H, Nakashima R, et al. Efficacy and safety of endoscopic submucosal dissection under general anesthesia. World J Gastrointest Endosc 2016; 8: 466-71.

3. Song BG, Min YW, Cha RR, Lee H, Min BH, Lee JH, et al. Endoscopic submucosal dissection under general anesthesia for superficial esophageal squamous cell carcinoma is associated with better clinical outcomes. BMC Gastroenterol 2018; 18: 80.

4. Lian J, Chen S, Zhang Y, Qiu F. A meta-analysis of endoscopic submucosal dissection and EMR for early gastric cancer. Gastrointest Endosc 2012; 76: 763-70.

5. Cao Y, Liao C, Tan A, Gao Y, Mo Z, Gao F. Meta-analysis of endoscopic submucosal dissection versus endoscopic mucosal resection for tumors of the gastrointestinal tract. Endoscopy 2009; 41: 751-7.

6. Park YM, Cho E, Kang HY, Kim JM. The effectiveness and safety of endoscopic submucosal dissection compared with endoscopic mucosal resection for early gastric cancer: a systematic review and metaanalysis. Surg Endosc 2011; 25: 2666-77.

7. Yagi Kuwata N, Gotoda T, Suzuki S, Mukai S, Itoi T, Moriyasu F. Reasonable decision of anesthesia methods in patients who underwent endoscopic submucosal dissection for superficial esophageal carcinoma: a retrospective analysis in a single Japanese institution. Turk J Gastroenterol 2016; 27: 91-6.

8. Heo HJ, Lee JH, Kim YY, Baek SM, Kim KM, Jung DW. Invisible perforation during an endoscopic procedure of the esophagus under general anesthesia - a case report. Anesth Pain Med (Seoul) 2020; 15: 383-7.

9. Lichtenstein DA. BLUE-protocol and FALLS-protocol: two applications of lung ultrasound in the critically ill. Chest 2015; 147: 1659-70. 
10. Isomoto H, Yamaguchi N, Minami H, Nakao K. Management of complications associated with endoscopic submucosal dissection/endoscopic mucosal resection for esophageal cancer. Dig Endosc 2013; 25 Suppl 1: 29-38.

11. Roberts DJ, Leigh-Smith S, Faris PD, Blackmore C, Ball CG, Robertson HL, et al. Clinical presentation of patients with tension pneumothorax: a systematic review. Ann Surg 2015; 261: 1068-78.

12. Raja AS, Jacobus CH. How accurate is ultrasonography for excluding pneumothorax? Ann Emerg Med 2013; 61: 207-8.

13. Denault A, Canty D, Azzam M, Amir A, Gebhard CE. Whole body ultrasound in the operating room and intensive care unit. Korean J Anesthesiol 2019; 72: 413-28.

14. Volpicelli G, Elbarbary M, Blaivas M, Lichtenstein DA, Mathis
G, Kirkpatrick AW, et al. International Liaison Committee on Lung Ultrasound (ILC-LUS) for International Consensus Conference on Lung Ultrasound (ICC-LUS). International evidence-based recommendations for point-of-care lung ultrasound. Intensive Care Med 2012; 38: 577-91.

15. Volpicelli G, Boero E, Stefanone V, Storti E. Unusual new signs of pneumothorax at lung ultrasound. Crit Ultrasound J 2013; 5: 10.

16. Loiselle A, Parish JM, Wilkens JA, Jaroszewski DE. Managing iatrogenic pneumothorax and chest tubes. J Hosp Med 2013; 8: 402-8.

17. Mavridis S, Gnauk HG, Schumacher M, Wagner R. Bilateral pneumothoraces complicating reduction mammoplasty: a case report. BMC Surg 2013; 13: 29. 